This is the author's manuscript for publication. The publisher-formatted version may be available through the publisher's web site or your institution's library.

\title{
A threatening exchange: gender and life history strategy predict perceptions and reasoning about sexual harassment
}

Haley Moss Dillon, Lora E. Adair, Gary L. Brase

\section{How to cite this manuscript}

If you make reference to this version of the manuscript, use the following information:

Dillon, H. M., Adair, L. E., \& Brase, G. L. (2015). A threatening exchange: Gender and life history strategy predict perceptions and reasoning about sexual harassment. Retrieved from http://krex.ksu.edu

\section{Published Version Information}

Citation: Dillon, H. M., Adair, L. E., \& Brase, G. L. (2015). A threatening exchange: Gender and life history strategy predict perceptions and reasoning about sexual harassment. Personality and Individual Differences, 72, 195-199.

Copyright: (C) 2014 Elsevier Ltd.

Digital Object Identifier (DOI): doi:10.1016/j.paid.2014.09.002

Publisher's Link: http://www.sciencedirect.com/science/article/pii/S0191886914005030

This item was retrieved from the K-State Research Exchange (K-REx), the institutional repository of Kansas State University. K-REx is available at http://krex.ksu.edu 


\title{
Final accepted manuscript of:
}

Dillon, H. M., Adair, L. E., \& Brase, G. L. (2015). A threatening exchange: Gender and life history strategy predict perceptions and reasoning about sexual harassment. Personality and Individual Differences, 72, 195-199.

\section{A threatening exchange: Gender and life history strategy predict perceptions and reasoning about sexual harassment}

\author{
Haley Moss Dillon ${ }^{1}$ \\ Lora E. Adair ${ }^{1}$ \\ Gary L. Brase ${ }^{1}$ \\ ${ }^{1}$ Kansas State University
}

Address for Correspondence: Gary L. Brase, Department of Psychological Sciences, 492 Bluemont Hall, Kansas State University, Manhattan, KS 66506, Email: gbrase@ksu.edu, Telephone: (785) 532-0609, Fax: (785) 532-5401

\begin{abstract}
Sexual harassment is a serious societal issue, with extensive economic and psychological consequences, yet it is also an ill-defined construct fundamentally defined in terms of subjective perception. The current work was designed to examine the ways in which individual differences between people are systematically related to different perceptions of sexual harassment scenarios, as well as reasoning about those harassment situations. Participants $(\mathrm{N}=460)$ read several possible harassment scenarios and rated how uncomfortable they would find them. They then also evaluated a quid pro quo sexual harassment situation in terms of their interpretation of it as a threat or a social exchange and completed a deductive reasoning task about the same situation. Females and individuals with slow life history strategies were more uncomfortable with potential harassment situations and were more likely to interpret the quid pro quo scenario as a threat. Further, interpreting the scenario as a threat was associated with poorer performance on the deductive logic task, compared to those who interpreted the scenario as a social exchange.
\end{abstract}

Keywords: Sexual Harassment; Decision Making; Social Exchange; Threats; Human Reasoning 


\section{Threatening exchange: Perception of sexual harassment predicts performance on Wason Selection Task}

Although human ability to reason in formal logic contexts tends to be poor, our ability to reason about certain social situations appears to be much better. A considerable body of literature now documents our abilities to reason well about with whom we should mate (Miller $\&$ Todd, 1998), who might pose a threat to our well-being and survival (Boyer \& Bergstrom, 2010), and whom we should trust in social interactions (Cosmides \& Tooby, 1992; Ekman, O’Sullivan, \& Frank, 1999). The current work examines decision making at the intersection of formal deductive logic and social reasoning. Specifically, our goal is to examine the ways in which people reason about sexual harassment situations, make decisions in such contexts, and how those responses compare to both normative formal logic standards and practical standards. Additionally, this work looks at individual differences in gender and in Life History Strategy (LHS) as factors influencing reasoning and decision making in this context.

\section{Why Sexual Harassment?}

Sexual harassment is broadly recognized as a phenomenon that has serious and pervasive implications for society, not only as a social issue but also as an economic, legal, and psychological well being issue (MacKinnon, 1979). Yet sexual harassment as a construct is quite "gray"; it is characterized by legal, political, and academic debate. The ambiguity of this definition has been critiqued in academic discourse, often citing findings which suggest that the "standpoint of a reasonable person" does not capture individual differences in the way that sexual harassment is perceived (Rotundo, Nguyen, \& Sackett, 2001). For example, females are more likely to perceive a broader range of behaviors as harassment (Rotundo, Nguyen, \& Sackett, 2001).

Little work, however, has systematically delineated how the subjectivity of this sexual harassment definition creates implications for how people reason and make decisions about sexual harassment. For this reason, we turn our attention in this research to this underexplored issue, and we employ a clear, objective substrate for our research design: human reasoning as studied in relation to formal logic.

\section{Human Reasoning about Social Contexts}

Humans are limited in their reasoning ability, based on the time and energetic constraints within which we must operate as decision makers (Gigerenzer \& Selten, 2002). These limitations are sometimes used to account for why people are notoriously bad at formal deductive reasoning (e.g., Evans, 2002; Funder, 1987). However, Cosmides (1989) discovered that when a formal deductive reasoning task, called the Wason Selection Task, is framed in terms of a social exchange, participants perform dramatically better. According to Cosmides (1989) this differential performance in reasoning based on the context of the problem supports an evolutionary perspective on human reasoning - that the mind has been shaped through natural selection and has therefore developed specific solutions to specific problems related to survival and reproduction. Social exchange is one such domain that has been critically important for our ancestors' survival and reproduction; individuals can solve significant adaptive problems (such as deciding with whom to cooperate) so that all parties can enjoy mutual benefits. Conversely, people can use information available to them and recognize if someone has cheated them in a social exchange, allowing for ending that relationship or even retaliatory responses (Cosmides, 1989; Cosmides \& Tooby, 1989). It is of utmost importance to be able to conclude whether other people in one's social group are going to be truthful and honest in social exchanges or whether 
they are going to cheat. Cosmides (1989) defines "cheating" in this context as "a violation of the rule established" (p. 197).

The Wason Selection Task (WST; Wason, 1968), which was used in the above research and is a staple of the human reasoning field, is a reasoning problem wherein a subject is required to see if a conditional rule of the form "If $P$ then $Q$ " has been violated in four instances that are relevant to that rule (where $P$ and $Q$ can be any information). These four instances (represented by cards) give the antecedent and the consequent being true or false $(P, \operatorname{Not} P, Q$, Not $Q)$. The participant is tasked with choosing which cards need to be turned over in order to test the truth of the statement (e.g., turning over the $P$ card would reveal either $Q$ or Not $Q$ ). Formal logic dictates that, given a statement of "If $P$, then $Q$," the correct choice to examine the truth of that statement is the cards " $P$ " and "Not $Q$ ". In less abstract terms, say the statement is "If a person is drinking alcohol, then that person is 21 or older" in this case, $P$ is "a person drinking alcohol", and Not $Q$ is "a person who is not 21 or older". The other cards (a person who is 21 or older [Q] and a person who is not drinking alcohol [Not $P$ ] do not provide information which can logically falsify the conditional rule.

For Cosmides (1989) and some subsequent researchers (e.g., Liberman \& Klar, 1996), better performance on the WST emerged because the content mapped onto evolved mechanisms which include reasoning processes. In the context of a social exchange, individuals' abilities to perform well on the WST was due to an ability to detect "cheaters": violations of social contracts. When viewed as a social contract, the conditional statement can be phrased as: "If you take the benefit $(P)$, then you pay the cost $(Q)$," and participants are tasked with enforcing this social contract.

The cost/benefit structure of the social contracts (Cosmides, 1989) create a potential for individual differences in reasoning performance that are based in people having different evaluations of what constitutes "costs" and "benefits." One such difference between people in the valuation of actions is the difference between males and females with regard to sexual activity. On average, males perceive more benefits to sexual activity, whereas females on average perceive higher costs to sexual activity. Given this very fundamental difference in perceptions of costs and benefits, Brase and Miller (2001) examined if there might be corresponding sex differences in the perception and reasoning about quid pro quo sexual harassment.

Quid pro quo ("something for something"; QPQ) harassment is a form of sexual harassment in which there is a solicitation of sexual compliance through promises of reward or threats of punishment (Fitzgerald \& Hesson-McInnis, 1989). The structure of QPQ harassment is amenable to being expressed as a conditional statement. Brase and Miller (2001) examined whether a quid pro quo harassment statement was perceived as a social exchange and if that interpretation influenced participants' reasoning performance on the WST. Brase and Miller presented participants with a statement such as "If you spend the night with me, I will give you a promotion" and asked participants whether this statement was a threat or a social exchange. Participants were then given the same statement within a Wason Selection Task and asked to pick the cards that need to be evaluated to determine if the statement has been violated. Participants who perceived the situation to be a threat performed worse on the reasoning task than those participants who perceived the situation to be a social exchange. Furthermore, Brase and Miller (2001) found that males, who in most conditions were more likely to perceive the statement to be a social exchange, outperformed females on the WST. These findings suggest 
that the extent to which individuals' perceive an event as a social contract, even an illegal sexual harassment event, shapes their reasoning about how to evaluate that event.

\section{Extending Prior Research}

The current work is designed to clarify and build on the findings of Brase and Miller (2001), further exploring differential perceptions of sexual harassment scenarios and how these differential perceptions might predict patterns of reasoning. In particular, this prior research found that sex differences in how people evaluated sexual harassment situations were inconsistent. One possible reason for this inconsistency is that there are within-sex individual differences also playing a role, such as Life History Strategy (LHS). Life History Strategy originated in evolutionary biology as a way to describe the selection of traits in an organism that represent the trade-off between quantity and quality of offspring (MacArthur \& Wilson, 1967), initially in comparisons across species. A "fast" life history strategy (wherein quantity of offspring is prioritized) involves producing more offspring and lower investment in individual offspring, whereas a "slow" life history strategy (wherein quality of offspring is prioritized) involves producing fewer offspring and greater investment in individual offspring. Humans as a species have a very slow LHS, but research has progressed to look at individual variations in LHS within the population.

Several research investigations of individual variations in LHS among humans have revealed some striking associations between LHS and changes in fertility rates (Hill \& Kaplan, 1999), age of menstruation onset (Ellis, 2004), and well as parental and romantic patterns of attachment (Figueredo, Vasquez, Brumbach, Sefcek, Kirsner, \& Jacobs, 2005). In humans, these individual differences in LHS can be operationalized as the allocation of resources to either reproductive efforts (relatively fast LHS) or somatic efforts (relatively slow LHS). Because organisms have limited resources to spend (e.g., energy, time, money), deciding how to invest them involves trade-offs (Dillon, Adair, \& Wang, 2013). In general, females employ slower life history strategies than males, but trade-offs exist both across the lifespan and as individual differences (within each sex) across the continuum from fast to slow LHS.

It is proposed that people with a slow LHS will be less likely to view the statement "if you spend the night with me, I will promote you" as a social exchange (and more likely to view it as a threat). A person employing a slow LHS should perceive a much higher cost/benefit ratio regarding this type of opportunistic sexual activity, which makes it a particularly unviable as a social exchange. In contrast, a person with a relatively fast LHS will be more likely to view their sexuality as a commodity that could be exchanged for other benefits (i.e., it has a lower cost/benefit ratio which makes it amenable to transactions) and would be more likely to view the statement as a social exchange.

Gender and LHS should also produce differences in perceptions of sexual harassment scenarios overall. It can be further hypothesized, however, that females should report more discomfort with sexual harassment scenarios than males (consistent with Parental Investment Theory; Trivers, 1972), as well as being less likely to view a possibly sexually harassing scenario as a social exchange rather than a threat. Additionally, it should be the case that individuals with slow LHS (male and female) will express more discomfort and be more likely to perceive the sexual harassment scenarios as a threat. Conversely, individuals with fast LHS, who are reserving less of their resources or efforts, will be less uncomfortable, and more likely to interpret the scenarios as a social exchange.

\section{Hypotheses}


H1: Females will report more discomfort with possible sexual harassment scenarios than will males.

$\mathrm{H} 2$ : Females will be more likely than males to perceive sexual harassment scenarios as threats (rather than as social exchanges).

H3: Slow LHS will be correlated with more discomfort with possible sexual harassment scenarios.

H4: Slow LHS will be correlated with a greater likelihood of perceiving sexual harassment scenarios as a threat (rather than as a social exchange).

H5: People who interpreted the sexual harassment scenario as a social exchange, rather than as a threat, will show a pattern of improved reasoning consistent with prior studies on social contract reasoning with the Wason selection task. In other words, interpreting the scenario as a social exchange will lead to more selections of the normatively correct $P$ and not $Q$ options.

H6: Males will outperform females on the Wason Selection Task.

\section{Methods}

$\underline{\text { Participants }}$

To develop a more diverse sample for this study, participants were recruited through General Psychology courses at a large Midwestern University (these participants received partial credit towards a course requirement), via Facebook, and via Amazon's Mechanical Turk (these participants received a small monetary payment). 460 participants (70\% female and 30\% male) completed the study.

Procedure and Materials

This study was performed via an online survey software. Participants were asked basic demographic questions and then were led to a page with five workplace vignettes that could be interpreted as sexual harassment (see Appendix A). Each scenario used a gender-neutral name and situation. For each vignette participants rated their comfort on a scale from 1 to 7 , with 1 being "extremely uncomfortable" and 7 being "extremely comfortable." Participants then filled out the Mini-K (Figueredo, 2007), which is an established brief measure of individual life history strategy (Figueredo, et al., 2005). Participants were then asked to rate the following statement as either a "threat" or a "social exchange":

You work in a large company, in an office that is run by a particular supervisor. Things seem to be going fine, although you do not have much information about the inner workings of the company. Your supervisor comes to your office, and tells you the following: 'If you spend the night with me, then I will give you a promotion.'

Finally, participants were given a Wason Selection Task based on the prior sentence. They were instructed to identify which of four "cards" (situations relevant to the conditional rule in the last sentence) they would need more information about in order to gauge whether the supervisor violated the statement in dealing with other coworkers. The situations were: 1) This person spent the night with the supervisor, 2) The supervisor did not promote this person, 3) The supervisor promoted this person, 4) This person did not spend the night with the supervisor. Participants were told they could choose as many or as few of the four situations that they felt was necessary to investigate in order to determine whether the supervisor may have violated the statement in the past.

\section{Results}

Responses across the five sexual harassment vignettes were averaged to create a single variable to indicate comfort or discomfort. The five vignettes did not violate assumptions of 
homoscedasticity and were not overly correlated, thus averaging to make one variable is statistically sound; $F_{\max }=1.4, p<.01$. As predicted, females reported significantly more discomfort across the sexual harassment vignettes $($ Mean $=2.84)$ than did males $($ Mean $=3.58$; $t(478)=-7.50, p<.001)$. The significant difference between mean male and female comfort levels supports H1, though it is important to note that means for both sexes were below the "neutral" level, indicating that both sexes reported being uncomfortable with the vignettes (See Table 1). As hypothesized, females also chose "threat" over "social exchange" much more than males in characterizing the QPQ conditional statement. A difference of proportions test found that this was a significant difference; $\mathrm{z}=5.48, p<.001, \eta=.54$, $(88.2 \%$ of females and $65.1 \%$ of males chose threat).

As predicted, Life History Strategy (LHS) correlated negatively with responses to the vignettes $(r=-.23, p<.01)$. Thus, slower LHS was associated with more uncomfortable reactions to the harassment scenarios. LHS was also correlated with the interpretation of a QPQ rule as a threat versus as a social exchange (SE), as hypothesized. Those with slower life history strategies were more likely to interpret the situation as a threat $\left.\mathrm{r}_{\mathrm{pb}}=.11, p=.02\right)$. Participants who interpreted the conditional rule as a threat had a higher Mini-K score (Threat $M=104.28$, $\mathrm{SE}=95.27)$. Gender was held constant for these analyses; furthermore, split-file analyses by sex yielded the same pattern of results. To further elucidate the potential relationship between sex, LHS, and comfort ratings we performed a two-way ANOVA using both sex and LHS as fixed factors and comfort ratings as our criterion. Consistent with our pattern of results, we observed main effects of both sex and LHS on comfort ratings but no interaction effects; for sex, $F(2$, $460)=26.204, p<.001$, for LHS, $F(2,460)=1.481, p<.05$.

To further interpret the relationship between sex, LHS, and perceptions of the conditional rule, a two-way ANOVA was run with perception of the conditional rule as the criterion and both sex and LHS as fixed factors. This was consistent with the previous results, suggesting that both sex and LHS significantly predict discomfort ratings and that there was an interaction between sex and LHS. Simple effects were examined for each sex (See Table 2). The simple effect for male participants $(F(2,448)=13.89, p<.05)$ indicates that male participants demonstrate varying perceptions of the scenario based on their LHS, such that males with slower LHS demonstrated a greater likelihood of perceiving the scenario as a threat. The simple effect for female participants $(F(2,448)=1.68, \mathrm{~ns})$ indicates that female participants did not demonstrate varying perceptions of the scenario based on their LHS, such that females with slower life history strategy tended to demonstrate a greater likelihood of perceiving the scenario as a threat, but this pattern was not significant. Across levels of life history strategy, females generally tended to perceive the scenario as a threat.

Brase and Miller (2001) found that when participants perceived a situation as a social exchange, rather than as a threat, they showed better reasoning performance on a Wason Selection Task (WST). Hypothesis 5 was based on this finding and replicated this result. Of participants who interpreted the conditional QPQ statement as a "threat", $11.7 \%$ correctly identified the logically correct $P$ and not $Q$ answers for the WST, whereas of those who interpreted the conditional QPQ statement as a "social exchange", 21.1\% correctly identified the answers (a significant increase; $\mathrm{z}=2.33, p=.01, \eta=.26$ ). Finally, Brase and Miller (2001) found that males outperformed females on the WST, which was the basis for our final hypothesis. This hypothesis was not supported by these data, although a trend consistent with it was present: more males $(12.8 \%)$ versus females $(8.6 \%)$ identified the normatively correct answers on the WST $(\mathrm{z}=1.38, p=.08, \eta=.14)$. 
Finally, regressions were run to determine whether sex and Life History Strategy were independently predicting perceptions of the conditional rule as well as reactions to the sexual harassment vignettes. A binary logistic regression analysis demonstrated that sex and LHS together accounted for $15 \%$ of the variance in how the QPQ conditional rules was interpreted $\left(\right.$ Nagelkerke $\left.\mathrm{R}^{2}=.15, \mathrm{~F}(2,448)=25.28, p<.001\right)$. Sex predicted interpretation of the conditional rule significantly $(\beta=1.18, p<.001)$, as did Life History Strategy $(\beta=-.03, p<$ .001). A simultaneous linear regression analysis demonstrated that sex and LHS together accounted for $13 \%$ of the variance in comfort ratings of harassment vignettes (Adjusted $\mathrm{R}^{2}=$ $.131, \mathrm{~F}(2,453)=35.40, p<.001)$. Sex predicted comfort on the sexual harassment vignettes $(B=$ $.31, p<.001)$, as did LHS $(\beta=-.14, p=.001)$. Collinearity statistics indicate that both sex and LHS were independently predicting reactions to the comfort ratings (tolerance $=.963, \mathrm{VIF}=$ 1.038).

\section{Discussion}

As predicted, females were more uncomfortable with possible sexual harassment scenarios and were more likely to perceive the quid pro quo sexual harassment situation as a threat, as opposed to a social exchange, compared to males. This is consistent with a relative difference between the sexes in valuing sexual access, as predicted by parental investment theory. In other words, because females have a greater minimum investment in offspring they place more value on sexual access, which leads them to be choosier regarding mates and more wary of those who are trying to gain sexual access.

Slower life history strategy was also found, as predicted, to correlate with more uncomfortable reactions to sexual harassment scenarios and with interpreting a QPQ sexual harassment situation as a threat. These results are consistent with slower LHS individuals having more focus on high investment in relatively few reproductive events. Indeed, individuals with slower LHS are more likely to obtain high quality mates (Dillon, Adair, Wang \& Johnson, 2013) and invest more effort in obtaining and maintaining relationships with high quality mates (Figueredo, Vasquez, Hagenah Brumbach, Sefcek, Kirsner, \& Jacobs, 2005) than in pursuing parenthood. Following this same logic, those with slower LHS are likely to make relatively affirmative decisions regarding possibly harassing scenarios (i.e., will be more likely to perceive ambiguous situations as being harassment). We made no specific hypotheses regarding whether LHS or sex would be a larger predictor variable, however split-file analyses tended to demonstrate the same pattern of effects, indicating that these constructs may be related but are generally independent. The one exception to this is the significant interaction identified between LHS and sex when predicting reactions to our sexual harassment scenarios - while both sexes tended to be more likely to perceive the scenario as a threat when their reported LHS was slower, this pattern of results was not significant for females. Across different life history strategies, females generally tended to perceive the scenario as a threat rather than as a social exchange.

Brase and Miller (2001) found that people who perceived the QPQ statement as a social exchange outperformed those who perceived the statement as a threat on the Wason Selection Task. The present study replicated this result. This lends credence to Cosmides's (1989) theory that people perform better on formal logic tasks when they are framed in a social exchange context, with the caveat that this framing can be partially determined by how the individual perceives the context. This suggests that legal definitions of harassment which rely on the "standpoint of a reasonable person" are likely not capturing individual variation in the perception of these social interactions. 
The one hypothesis not fully supported in the present study was a sex difference in Wason selection task performance, based on the results of Brase and Miller (2001). The current study found a trend such that more males correctly answered the WST than females, but this difference was not significant. It is possible that this non-significance could be due to the relatively low percentage of correct WST answers and that an overall better performance rate would have led to clearer results. This relatively low number of correct ( $P$ and not $Q$ ) responses for the Wason Selection Task may be an issue of concern. Only 13.3\% of the total sample correctly chose the $P$ and not $Q$ responses, which are the normatively correct responses (according to formal logic) and the correct responses in terms of a social exchange (corresponding to "benefit taken" and "cost not paid") for determining if someone has "cheated" or violated a stated social rule. Because the percentage of correct responses is so low (compared to $23.5 \%$ correct responses in Brase and Miller's 2001 study), it is possible that we may have missed relationships that would have shown up with a larger percentage of correct WST answers. It is unclear at this point whether the low performance is due to participants' inability to correctly identify the answers or due to something such as a misunderstanding of directions. Future studies need to take measures to clarify this aspect of the present results.

One implication of the current work is that the lower performance on the Wason Selection Task of those who did not categorize the QPQ statement as a social exchange may not be exclusive to logic tasks. It is possible that feeling threatened may lead to decreased ability to make decisions. Some work does suggest that emotional arousal can contribute to experiences of bounded rationality - that is, when an individual is experiencing high levels of emotional arousal their ability to contribute cognitive resources to rational decision-making is compromised (Kaufman, 1999). As Loewenstein and Lerner (2003) explain, “As emotions intensify, they exert an ever-increasing influence on behavior. Indeed, at sufficient levels of intensity, emotions can overwhelm cognitive processing and deliberative decision making altogether" (pg. 627). We believe that interpreting social exchanges as threatening may affect reasoning and decision making beyond the reasoning performance measured in the current study. It is important to further our understanding of how feeling threatened in various social contexts (e.g., at work, at home, at school) may lead to impaired decision making ability. For example, it is possible that feeling threatened may lead to deficiencies in work performance and therefore an increased likelihood of being exploited in this social context. Additionally, sexual harassment scenarios (regardless of their ambiguity) may have a different reaction than other social exchanges. However, as this work primarily aimed to replicate and extend Brase and Miller (2001), we used scenarios only related to sexual harassment. Future research would benefit from similar methodology using different types of threats.

The current work provides an initial insight into how our reasoning ability might be shaped by our subjective perceptions of social interactions. Future work should expand on our current findings by perhaps exploring additional individual difference factors that might predict how one interprets various social interactions and exchanges, and perhaps if these perceptions are shaped by the context in which they are encountered.

\section{References}

Boyer, P., \& Bergstrom, B. (2011). Threat-detection in child development: An evolutionary perspective. Neuroscience \& Biobehavioral Reviews, 35, 1034-1041.

Brase, G.L. \& Miller, R.L. (2001). Differences in the perception of and reasoning about quid pro quo sexual harassment. Psychology, Evolution \& Gender, 3, 241-264. 
Cosmides, L. (1989). The logic of social exchange: Has natural selection shaped how humans reason? Studies with the Wason selection task. Cognition, 31, 187-276.

Cosmides, L., \& Tooby, J. (1989). Evolutionary psychology and the generation of culture, Part II. Case study: A computational theory of social exchange. Ethology and Sociobiology, 10, 51-97.

Cosmides, L., \& Tooby, J. (1992). Cognitive adaptations for social exchange. In: J. H. Barkow, L. Cosmides, \& J. Tooby (Eds.), The Adapted Mind: Evolutionary Psychology and the Generation of Culture (pp. 163-228). London: Oxford University Press.

Dillon, H.M., Adair, L.E., Wang, Z. \& Johnson, Z. (2013). Slow and steady wins the race: Life history, mate value, and mate settling. Personality and Individual Differences, 55, 612618.

Ekman, P., O’Sullivan, M., \& Frank, M.G. (1999). A few can catch a liar. Psychological Science, 10, 363-366.

Ellis, B. J. (2004). Timing of pubertal maturation in girls: an integrated life history approach. Psychological Bulletin, 130, 920-958.

Evans, J. S. B. (2002). Logic and human reasoning: an assessment of the deduction paradigm. Psychological Bulletin, 128, 978-996.

Figueredo, A.J. (2007). The Arizona Life History Battery. Retrieved from: http://www.u.arizona.edu/ ajf/alhb.html

Figueredo, A. J., Vasquez, G., Brumbach, B. H., Sefcek, J. A., Kirsner, B. R., \& Jacobs, W. J. (2005). The K factor: Individual differences in life history strategy. Personality and Individual Differences, 39, 1349-1360.

Funder, D. C. (1987). Errors and mistakes: evaluating the accuracy of social judgment. Psychological Bulletin, 101, 75-90.

Gigerenzer, G., \& Selten, R. (Eds.). (2002). Bounded Rationality: The Adaptive Toolbox. Cambridge, MA: MIT Press.

Hill, K., \& Kaplan, H. (1999). Life history traits in humans: Theory and empirical studies. Annual Review of Anthropology, 28, 397-430.

Kaufman, B. E. (1999). Emotional arousal as a source of bounded rationality. Journal of Economic Behavior \& Organization, 38(2), 135-144.

Liberman, N., \& Klar, Y. (1996). Hypothesis testing in Wason's selection task: Social exchange cheating detection or task understanding. Cognition, 58, 127-156.

Loewenstein, G., \& Lerner, J. S. (2003). The role of affect in decision making. In R. J. Davidson, K. R. Scherer, \& H. H. Goldsmith (Eds.) Handbook of Affective Science (619-642). New York, NY: Oxford University Press.

MacArthur, R.H. \& Wilson, E.O. (1967). The theory of island biogeography. Princeton University Press, Woodstock, OX.

MacKinnon, C.A. (1979). Sexual Harassment of Working Women. New Haven: Yale University Press.

Miller, G. F., \& Todd, P. M. (1998). Mate choice turns cognitive. Trends in Cognitive Sciences, 2, 190-198.

Rotundo, M., Nguyen, D. H., \& Sackett, P. R. (2001). A meta-analytic review of gender differences in perceptions of sexual harassment. Journal of Applied Psychology, 86, 914922.

Trivers, R.L. (1972). Parental investment and sexual selection. In B. Campbell 
(Ed.), Sexual Selection and the Descent of Man, 1871-1971 (pp. 136-179). Chicago, IL: Aldine.

Wason, P. C. (1968). Reasoning about a rule. The Quarterly Journal of Experimental Psychology, 20, 273-281.

\section{Appendix A}

Alex heads the mail room at a large insurance company. Alex is in charge of many new hires, including you, who are anxious to move ahead in the company. One day, Alex says to you, "Why don't we meet for drinks tonight to celebrate your new promotion." You have not been told about getting a promotion yet, so you are anxious about what is involved in "meeting for drinks."

Sam is your manager. You are working together on a project and Sam has asked you to dinner to discuss your work. After dinner, Sam says "perhaps if we cooperate well on this project, I can make things easier for you at your next evaluation." You think that Sam's tone of voice seems to suggest that by "cooperate" Sam means something sexual.

Mel does a lot of research on the internet. Occasionally Mel finds funny jokes or photos, some of them off-color, that Mel likes to email to coworkers. Most of the coworkers find these jokes a welcome break. You, however, find them offensive and would like Mel to stop sending them. When you ask Mel to stop, some co-workers think you should "lighten up."

You work in a graphic arts department, and are the only person of your gender in the office. Every day the other employees in the office await the arrival of a very attractive delivery person. After the delivery person leaves, the other employees spend several minutes making suggestive remarks and jokes about the person's attractiveness.

During a meeting, your boss says, "Why don't you wear more attractive outfits like those lawyers on TV. You're an attractive person; I think if you showed yourself off a little, the clients would be happier and I'd be happier. A little dedication on your part could go a long way here." 